\title{
Lesbos, l'île aux grillages. Migrations et enfermement à la frontière gréco-turque
}

Lesbos, the Island of Barriers. Migration and Containment at the Greece-Turkey Border

\section{Laurence Pillant et Louise Tassin}

\section{(2) OpenEdition}

\section{Journals}

\section{Édition électronique}

URL : http://journals.openedition.org/conflits/19068

DOI : 10.4000/conflits. 19068

ISSN : 1777-5345

Éditeur :

CECLS - Centre d'études sur les conflits - Liberté et sécurité, L'Harmattan

Édition imprimée

Date de publication : 31 décembre 2015

Pagination : 25-55

ISBN : 978-2-343-08728-3

ISSN : 1157-996X

Référence électronique

Laurence Pillant et Louise Tassin, « Lesbos, I'île aux grillages. Migrations et enfermement à la frontière gréco-turque », Cultures \& Conflits [En ligne], 99-100 | automne/hiver 2015, mis en ligne le 16 février 2017, consulté le 31 mars 2021. URL : http://journals.openedition.org/conflits/19068 ; DOI : https:// doi.org/10.4000/conflits. 19068 


\section{Lesbos, I'île aux grillages. Migrations et enfermement à la frontière gréco-turque}

\section{Laurence PILLANT et Louise TASSIN}

Laurence Pillant est doctorante en géographie à l'UMR TELEMMe (Aix Marseille Université) et à l'Université de Harokopio (Athènes). Ses travaux portent sur les transformations socio-spatiales inbérentes aux développements du contrôle migratoire à la frontière gréco-turque à partir du XXIe siècle. Elle a notamment publié "Les conséquences socio-spatiales des nouvelles modalités du contrôle migratoire à la frontière gréco-turque", L’Espace Politique, n²5, avril 2015, en ligne, et "Introduction of procedures for migration controls in Greece. Case study in the Aegean Islands", in Anteby L., Baby-Collin V., Mazzella S., Mourlane S., Parizot C., Regnard C., Sintès P. (collectif MIMED) (eds.), Borders, Migrations and mobilities. Perspectives from the Mediterranean, Peter Lang, 2014.

Louise Tassin est doctorante en sociologie à l'URMIS (Université Nice Sophia Antipolis). Ses travaux portent sur l'enfermement administratif des étrangers en instance d'expulsion dans l'Union européenne à travers une étude de cas en région parisienne, au sud de la Sicile et à la frontière gréco-turque. Elle a notamment publié "Accueillir les indésirables. Les habitants de Lampedusa à l'épreuve de l'enfermement des étrangers", Genèses, 96, septembre 2014, et " "Ne cassez pas ce qui vous profite". Le rôle ambivalent des objets dédiés au confort en situation d'enfermement ", Champ pénal/Penal field, vol. XI, 2014, en ligne.

Située en mer Égée à une douzaine de kilomètres des côtes turques, l'île grecque de Lesbos a été au cœur de l'actualité européenne pendant l'été $2015^{1}$ au cours duquel des milliers de migrants ${ }^{2}$ ont réussi à gagner clandesti-

1. Quelques exemples, parmi d'autres, dans la presse : Le Monde, «Lesbos, l'île au bord du naufrage ", 17 juin 2015; Libération, "Sur l'île de Lesbos, le défi du secteur touristique face aux migrants », 27 septembre 2015 ; New York Times, "On Island of Lesbos, a microcosm of Greece’s other crisis : migrants », 4 août 2015.

2. Bien que se trouvent parmi les personnes débarquées à Lesbos des individus susceptibles d'obtenir le statut de « réfugié » tel que défini par la Convention de Genève de 1951, nous lui préférons le terme générique, moins normatif et plus descriptif de « migrants » pour désigner, sans distinction a priori, celles et ceux qui se trouvent, sur l'île de Lesbos, en situation de migration. Ce choix, s’il ne doit pas masquer que les personnes ainsi désignées fuient pour 
nement l'île par la mer ${ }^{3}$. Leur nombre a donné à ces arrivées une dimension spectaculaire : 445037 personnes ont été interceptées durant les onze premiers mois de l'année 2015 contre 12187 l'année précédente et 13171 en 2008, chiffre jusque-là le plus élevé 4 . Dépassées, les autorités locales ont réagi dans l'urgence en recourant à ce qui est devenu, depuis les années 2000, le ressort principal de la lutte contre l'immigration clandestine dans l'Union européenne (UE) : les lieux de rétention administrative ${ }^{5}$. Les étrangers ont été placés dans un centre destiné au tri et à l'expulsion des personnes en situation irrégulière puis, faute d'espace suffisant, dans des camps de fortune installés dans la précipitation en divers endroits de l'île. Très vite surpeuplés et insalubres, ces lieux ont fait l'objet de vives critiques de la part des acteurs locaux, des organisations internationales et des $\mathrm{ONG}$ présentes sur place ${ }^{6}$.

Tout en étant exceptionnelle par son ampleur, cette situation n'est cependant pas surprenante pour qui connaît l'histoire de l'île où, depuis une dizaine d'années, l'urgence est devenue chronique et l'improvisation la règle en matière de contrôle migratoire.

beaucoup des situations de guerre, est selon nous nécessaire pour deux raisons : d'une part, qualifier unanimement les migrants de "réfugiés » tend à occulter le fait que ce statut, précisément, n'est accordé qu'à un nombre marginal de personnes dans l'UE (Agier M., «Exilés, réfugiés, déplacés, déboutés...Vers un monde sans asile ? ", in Migreurop, Atlas des migrants en Europe, Paris, Armand Colin, 2012, pp. 16-19) ; d'autre part, il participe d'une vision dichotomique des migrations fondée sur l'opposition entre migrants économiques et demandeurs d'asile qu'il nous semble important d'interroger et de déconstruire. Sur cette question, voir notamment : Morice A., Rodier C., "Classer-trier migrants et réfugiés : des distinctions qui font mal », Hommes et Libertés - Revue de la Ligue des Droits de l'Homme, 129, 2005, pp. 58-61 ; «La distinction entre réfugiés et migrants économiques ne va pas de soi », entretien avec Karen Akoka, Alterecoplus, 11 septembre 2015.

3. Ces arrivées ne doivent pas masquer les naufrages relativement fréquents dans cette zone : 84 personnes ont péri entre le 7 juillet et le 15 septembre 2015 en mer Egée et 24 personnes sont portées disparues, chiffre a priori inférieur à la réalité dans la mesure où il ne comptabilise que les personnes retrouvées par les autorités ou déclarées disparues par les proches. Ces chiffres sont issus du projet The Migrants Files [http://www.themigrantsfiles.com].

4. Ces chiffres sont disponibles sur le site internet de la police grecque [http://www.astynomia.gr].

5. Nous choisissons d'utiliser le terme français de « rétention » pour désigner les lieux utilisés pour enfermer des étrangers en situation irrégulière à des fins de tri ou d'expulsion, que ces espaces soient officiellement destinés ou non à cet effet. Cette notion n'a pas de traduction littérale en grec ou en anglais et tend à euphémiser la dimension carcérale des dispositifs concernés. Elle permet cependant de souligner le caractère administratif et non pénal de la privation de liberté, rappelant ainsi la spécificité de cette forme exceptionnelle de confinement extra-judiciaire. Le terme « détention » sera quant à lui utilisé de façon générique pour évoquer le maintien dans une structure fermée.

6. Voir notamment les communiqués de presse de Médecins sans frontières [http://www.msf.org.uk/article/greece-msf-urges-action-on-the-reception-crisis-faced-bythousands] et le rapport d'un volontaire de l'UNICEF [http://www.statewatch.org/news/2015/aug/greece-camp-Kara-Tepe-report-andproposals.pdf] qui dénoncent l'insalubrité du camp de Kara Tepe (Ka@ó Teлć) installé dans l'urgence dans un circuit d'auto-école. Des articles de la presse locale relatent également les témoignages de riverains qui critiquent le caractère durable de cette solution censée être temporaire [http://www.emprosnet.gr/article/74687-katastimatarhes-kara-tepe-sto-dimarhoepiliftheite-tis-katastasis-tora]. 
Confrontée à un nombre important, quoique relatif 7, d'entrées irrégulières sur son territoire (figure 1), la Grèce a en effet adopté principalement des mesures répressives à l'égard des migrations : avec le soutien matériel et financier de l’Union européenne, le pays s'est engagé à partir des années 2000 dans un processus de sécurisation de ses zones frontalières terrestres (région d'Evros) et maritimes (îles de la mer Egée) ${ }^{8}$ dont l'enfermement est devenu la pierre angulaire. Phénomène généralisé dans l'UE et au-delà 9 , la rétention des étrangers s'est imposée comme le mode de gestion principal des arrivées de migrants, entraînant sur l'île l'utilisation et la création de lieux hybrides caractérisés par un régime d'exception peu à peu institutionnalisé ${ }^{10}$.

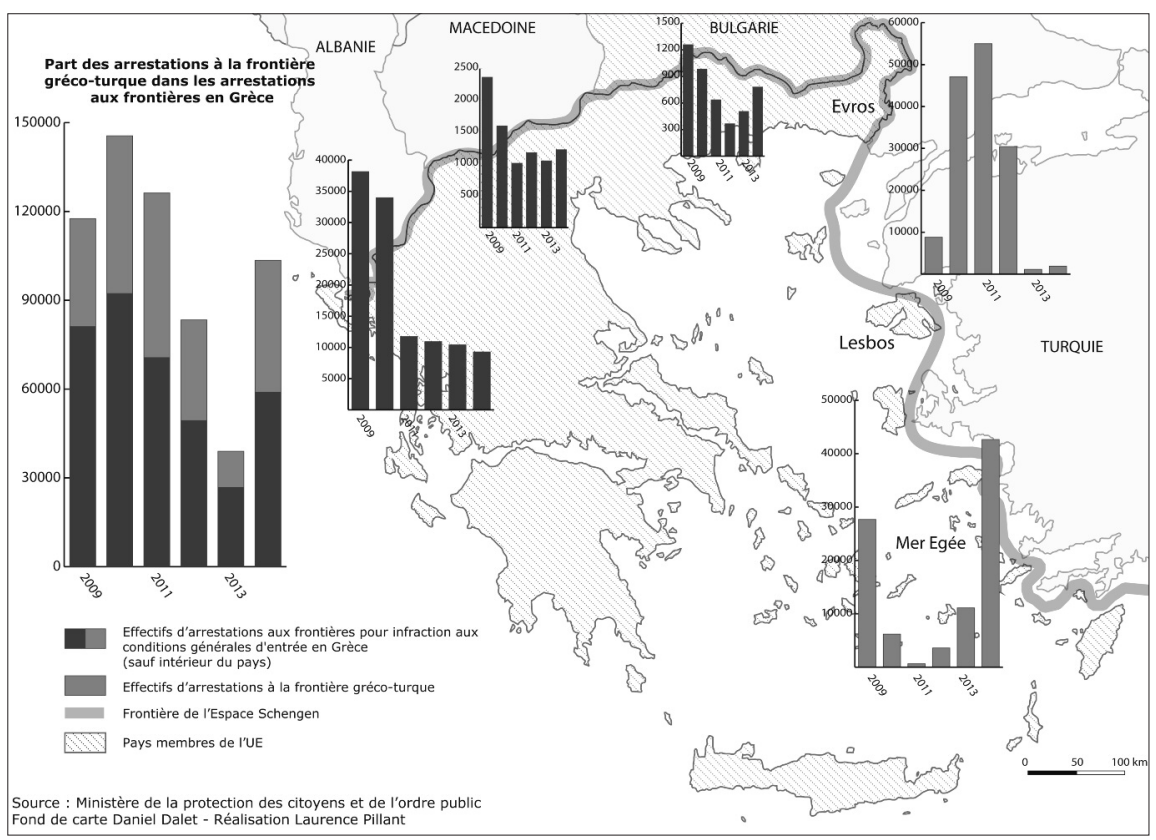

Figure 1

7. Ces chiffres gagneraient à être mis en perspective avec l'histoire de l'immigration dans l'UE et les réalités statistiques des mouvements de personnes à l'échelle mondiale. En 2013, $36 \%$ des migrations internationales ont lieu entre les pays du Sud alors que $35 \%$ suivent un axe Sud-Nord. Parmi ces dernières, $40 \%$ ont l'Europe comme destination, ce qui représente seulement $13 \%$ des migrations internationales (Rapport du département des affaires sociales et économiques des Nations Unies n ${ }^{\circ} 2013 / 3$, avril 2014). En 2013, la Grèce comptabilise 9 \% des « ressortissants de pays tiers repérés en situation illégale " dans l'UE, ce qui, rapporté à l'échelle nationale, ne représente que 0,36 \% de sa population (Eurostat [migr_eipre] 2013). Tous n'ont par ailleurs pas franchi les frontières clandestinement.

8. Topak O. E., "The biopolitical border in practice: surveillance and death at the GreeceTurkey boderzones", Environment and Planning D: Society and space, 32/5, 2014, pp. 815833.

9. Massa E., Silverman SJ., "Why Immigration Detention Is Unique", Population, Space and Place, 18/6, 2012, pp. 677-686; Valluy J., "Introduction : L'Europe des camps. La mise à l'écart des étrangers ", Cultures E Conflits, 57, 2005, pp. 5-11.

10. Sur les processus d'institutionnalisation de l'exception, voir : Fischer N., «Un lieu d'exception ? Retour sur le statut de la rétention administrative dans un contexte démocratique », Politix, 4/104, 2013, pp. 181-201. 
Comment ces derniers ont-ils été choisis, transformés, abandonnés, réutilisés, construits, et dans quelle mesure cette évolution a-t-elle façonné la vie locale ? Le cas de Lesbos, plus grande île de la frontière gréco-turque devenue au fil des ans un espace clef du contrôle des migrants à l'échelle nationale et européenne, offre un terrain privilégié pour observer le développement de l'enfermement des étrangers aux confins de l'UE. Emblématique du «blindage » des frontières extérieures de l'Union européenne ${ }^{11}$ et de ses ambivalences, il permet d'analyser les processus de construction et de contestation des dispositifs formels et informels de confinement des migrants, questionnant ainsi certains effets et limites du recours à la rétention.

Si les centres de rétention administrative ont fait l'objet d'une littérature croissante dans la dernière décennie ${ }^{12}$, la question de leur impact sur les territoires où ils sont installés reste encore peu abordée. Maintenus dans une relative opacité, ces lieux difficiles d'accès à la société civile, aux journalistes et aux chercheurs ont été surtout étudiés pour leur fonctionnement interne, avec des travaux consacrés aux ressorts du contrôle, aux relations entre les différents corps professionnels et les étrangers retenus et aux éventuelles marges de manœuvre de ces derniers. Forts de cette littérature, plusieurs travaux récents ont souligné l'importance de « décloisonner l'enfermement » pour s'intéresser à certaines formes de circulation contrainte et dépasser ainsi les dichotomies dedans/dehors ou ouverture/fermeture ${ }^{13}$, invitant à penser les lieux d'enfermement au-delà des "murs » qui les délimitent ${ }^{14}$. Dans le prolongement de ces recherches, nous proposons d'examiner, à travers le cas de Lesbos, les relations entre les lieux de rétention et leur environnement. Cela implique, d'une part, d'examiner la genèse et les implications de leur installation, en partant de l'hypothèse que les centres de rétention, structures fermées et isolées par excellence, conditionnent et sont conditionnés par le tissu social dans lequel

11. Ritaine E., «La fabrique politique d'une frontière européenne en Méditerranée, le jeu du Mistigri entre l'État et l'Union européenne », Les études du CERI, 186, juillet 2012.

12. Clochard O., "Les étrangers à Chypre, entre enfermement et mise à l'écart » in Kobelinsky C., Makaremi C., (eds.), Enfermés dehors. Enquetes sur le confinement des etrangers, Paris, Editions du croquant, 2009, pp. 281-297 ; Darley M., «L'enfermement des etrangers en Autriche et en Republique tchèque : du controle et de son contournement », in Kobelinsky C., Makaremi C., ibid., pp. 63-84 ; Fischer N., "Entre urgence et contrôle », Recueil Alexandries, Collections Esquisses, 2007 [http://www.reseau-terra.eu/article560.html] ; Michalon B., "La mobilité au service de l'enfermement ? ", Géographie et cultures, 81, 2012, pp. 91-110 ; Martin L. L., Mitchelson M. L., "Geographies of detention and imprisonment: interrogating spatial practices of confinement, discipline, law, and state power”, Geograpy compass, 3/1, 2009, pp. 459-477 ; Massa E., Silverman S. J., "Why immigration detention is unique", Population, Space and Place, 18/6, 2012, pp. 677-686 ; Moran D., Gill N. et Conlon D., Carceral spaces, Mobility and Agency in Imprisonment and Migrant detention, Ashgate edition, 2013.

13. Kobelinsky C., Makaremi C. (eds.), op. cit. ; Kobelinsky C., Makaremi C. (eds.), "Confinement des étrangers: entre circulation et enfermement », Cultures E Conflits, 71, 2008 ; Akoka K., Clochard O., "Régime de confinement et gestion des migrations sur l'île de Chypre ", L'Espace politique, 1/25, mis en ligne le 5 avril 2015 [http://espacepolitique.revues.org/3381].

14. Darley M., Lancelevée C., Michalon B., «Où sont les murs ? Penser l'enfermement en sciences sociales », Cultures $E$ Conflits, 90, 2013, pp. 12-13. 
ils s'inscrivent. D'autre part, cela exige de prendre en considération d'autres lieux de confinement moins officiels avec lesquels ils fonctionnent (ports, fourgons, bateaux, restaurants, etc.) ${ }^{15}$, souvent absents de l'analyse parce que plus difficiles à approcher empiriquement ou considérés comme marginaux ${ }^{16}$. L'enjeu est de prêter ainsi attention aux marges du dispositif, à ce qui se passe en amont et en aval du placement dans une structure consacrée, qui nous en dit parfois tout autant, sinon plus, sur le fonctionnement du système.

Le cas de Lesbos est particulièrement intéressant dans cette perspective de recherche. Son caractère insulaire, son emplacement géographique et l'irrégularité des arrivées de migrants, massives ou marginales selon les périodes (figure 2), permettent d'étudier les recompositions du paysage frontalier au gré des flux migratoires, au cours mais aussi en dehors des périodes d'urgence et de médiatisation. Par ailleurs, l'enfermement s'y décline sous différentes formes, reflétant la diversité des situations observables dans l'UE : l'île a expérimenté plusieurs lieux de rétention, plus ou moins officiels, créés ou non à cet effet (commissariat, prison, hangar, préfabriqués, port); elle a connu diverses modalités d'enfermement, des plus strictes aux plus souples (centre de rétention, lieux ouverts, structures fermées dites d'accueil, campements provisoires); elle a été, enfin, le lieu d'initiatives locales alternatives à la détention, avec des projets lancés par des habitants que les autorités ont cherché à contrôler, voire à s'approprier. Tous ces espaces, chargés historiquement, pour certains abandonnés, pour d'autres encore en activité, permettent de saisir quelques tâtonnements, contradictions et implications de la systématisation du recours à la rétention aux frontières extérieures de l'UE.

Figure 2

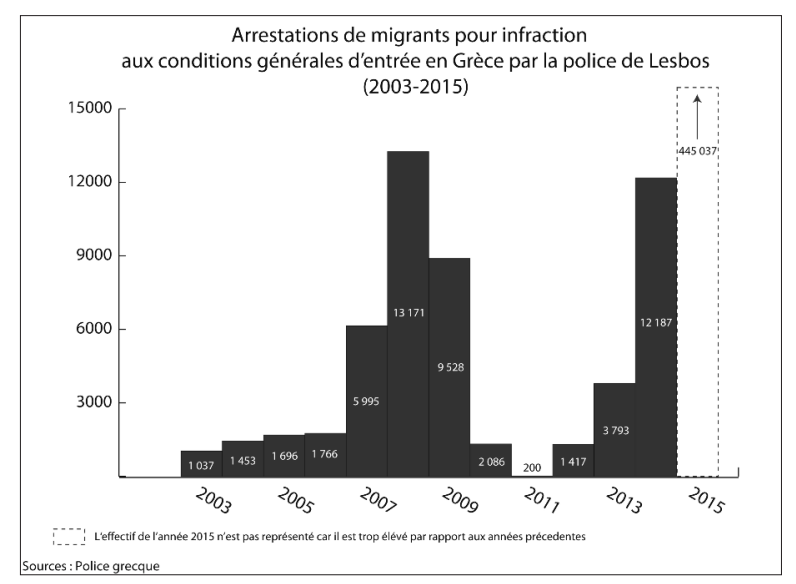

15. Le terme de confinement, qui comporte à la fois l'idée de réclusion et de voisinage (Akoka, K., Clochard O., op. cit.) se prête particulièrement à la désignation d'espaces liminaires dans lesquels les migrants sont enfermés et circulent à la fois, notamment les ports et commissariats.

16. Sur l'intérêt de l'étude des postes-frontières par exemple, voir : Darley M., « Frontière, asile et détention des étrangers. Le contrôle étatique de l'immigration et son contournement en Autriche et en République tchèque », thèse de science politique, IEP de Paris, 2008 ; sur les 
Après avoir montré comment, par-delà leur diversité et leur éclatement géographique, les différents lieux créés ou utilisés à des fins de rétention dessinent à Lesbos une forme de continuum placé sous le signe de l'urgence, nous verrons de quelle manière ce dispositif façonne et se trouve façonné par la société locale et ses habitants qui, opposés ou non à la détention des étrangers, employés ou non dans les lieux de rétention, sont mis à l'épreuve de l'enfermement.

\section{Des lieux de rétention disparates gérés dans l'improvisation}

De la fourgonnette au centre créé ad hoc, trois types d'espaces ont servi et servent encore à enfermer les étrangers qui débarquent sur l'île : des structures consacrées, des dispositifs de contrôle détournés de leur fonction et des locaux informels allant du restaurant privé au parc municipal. L'étude de ces lieux pris dans leur ensemble et observés dans la durée montre que la rétention ne se limite pas aux centres officiels mais prend corps dans une série de pratiques et d'espaces différents qui, allant de l'enfermement à certaines mobilités imposées, forment les maillons d'une chaîne de contrôle aux contours flous marquée par l'improvisation.

\section{Les dispositifs officiels : l'institutionnalisation de l'exception}

Si les premiers débarquements de migrants en provenance d'Afrique, d'Asie et du Moyen-Orient datent à Lesbos des années 1990, ce n'est qu'au milieu des années 2000 que le phénomène prend réellement de l'ampleur avec l'accélération des arrivées (figure 2). Après quelques années de gestion chaotique des étrangers au port et au commissariat, des membres de la société civile proposent de les réunir dans un espace désaffecté : une ancienne prison pour opposants politiques fermée dans les années 1960 puis transformée en service administratif. Situé en périphérie de la capitale, cet établissement se prête particulièrement à la rétention : utilisée pendant quelques temps comme une structure ouverte où les migrants étaient libres d'aller et venir, la prison devient progressivement un lieu de privation de liberté sous la pression notamment du voisinage qui, face à l'augmentation du nombre de migrants, fait part de ses craintes aux autorités. Apparaît ainsi le premier lieu officiel d'enfermement des étrangers sur l'île, rattaché à l'autorité religieuse orthodoxe de Mytilène ${ }^{17}$ mais géré par la préfecture qui, en pérennisant une solution d'urgence, pose la première pierre de ce qui va devenir un dispositif de rétention hybride : éclaté, protéiforme et discontinu mais peu à peu institutionnalisé. Les conditions de rétention dans l'ancienne prison ne sont cependant pas satisfaisantes (insalubrité, problèmes de chauffage, équipements insuffisants, etc.) et les révoltes se multiplient en son sein, suscitant l'inquié-

«lieux invisibles » de l'enfermement, voir plus généralement le travail du réseau Migreurop: [http://www.migreurop.org/]. 
tude des habitants des alentours. Les autorités décident alors dans la précipitation de fermer l'ancienne prison et de transférer les étrangers dans un entrepôt de marchandises dans le quartier de Pagani, nom qui deviendra bientôt le symbole de la maltraitance des migrants.

Cette décision s'inscrit dans un contexte de pression croissante en matière d'immigration à l'échelle nationale et européenne. Non seulement la Grèce, après les attentats du 11 septembre 2001, cherche à sécuriser le pays en vue des Jeux Olympiques d'août 2004, mais la mise en application des accords de Dublin II en 2003 (qui prévoient que les demandeurs d'asile interpellés dans l'espace Schengen soient renvoyés dans le premier pays où ils ont été identifiés) conduit le gouvernement à poser les bases du contrôle migratoire : instauration d'un cadre juridique spécifique à l'enfermement, fichage systématique des demandeurs d'asile et développement de lieux de confinement (loi $3386 / 2005)^{18}$.

Alors que le centre de Pagani est pensé comme une solution temporaire au point d'être géré par une division de la préfecture nommée « plan civil d'ur-

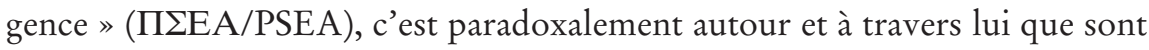
établies de manière durable les procédures relatives au contrôle des étrangers sur l'île. Le financement du centre est notamment confié au ministère de l'Intérieur tandis que l'absence d'anticipation - « Tout était justifié par l'urgence 19 »-contribue à la dégradation des conditions de rétention : cellules collectives surchargées, sanitaires insuffisants ou inutilisables, accès à une cour extérieure limité pour certains à quelques heures par mois, etc. ${ }^{20}$ Le centre, auquel sont ajoutés des préfabriqués au fur et à mesure, se développe au jour le jour sans que ne soit jamais envisagée sa transformation structurelle, pourtant moins onéreuse et nécessaire d'un point de vue juridique. Paroxysme de l'exception qui préside au traitement des migrants, Pagani sera finalement fermé en 2009 sous l'effet conjoint de mobilisations locales et de préoccupations nationales et européennes ${ }^{21}$ avant d'être utilisé pendant quelques mois pour les rares migrants qui débarquent sur l'île. Si la gestion discrétionnaire du lieu aboutit donc à sa dénonciation puis son abandon, elle consolide parallèlement le système d'enfermement.

17. Située au sud-est de l'île, Mytilène est la principale ville et le cœur économique de Lesbos.

18. Pillant L., "Les conséquences socio-spatiales des nouvelles modalités du contrôle migratoire à la frontière gréco-turque ", L'Espace politique, 1/25, mis en ligne le 5 avril 2015 [http://espacepolitique.revues.org/3381].

19. Entretien avec le secrétaire général en charge de la politique migratoire pour le ministère de l'Intérieur, février 2011, Athènes, LP.

20. Pro Asyl, "The truth may be bitter but it must be told", octobre 2007 et "The situation in Greece is out of control”, octobre 2008 ; Human Rights Watch "Stuck in a revolving door", décembre 2008 ; MSF, “Lives Hold”, juin 2010 ; Comité pour la prévention contre la torture (CPT), rapports 2005, 2007 et 2008.

21. Lauth Bacas J., "No safe haven: the reception of irregular boat migrants in Greece", Ethnologia Balkanica, n¹4, 2010, pp. 147-168. 
Par la suite, la diminution des flux migratoires fait passer au second plan les préoccupations liées à la migration. Après un projet de centre évoqué en 2010 et rapidement abandonné, il faut attendre septembre 2013 pour que le premier lieu construit officiellement à des fins de rétention voie le jour sur l'île. Son ouverture, anticipée sous la pression de la société civile, répond à des exigences nationales et européennes. Après des décennies de gestion plus ou moins improvisée des questions d'immigration, la Grèce vote en 2011 la première grande loi en la matière - 3907/2011 22 - qui transpose la directive européenne dite « retour» de $2008{ }^{23}$. Elle prévoit, entre autres, l'ouverture de «centres de premier accueil » (KEPY) aux frontières du pays, le développement de l'enfermement à des fins d'expulsion et l'allongement de la durée maximale de rétention à 18 mois, limite autorisée dans l'UE. Le centre de Lesbos, installé dans la commune de Moria, appartient à la première catégorie. Situé dans une ancienne zone militaire, composé de préfabriqués encadrés de grillages, de barbelés et de guérites, il doit officiellement servir à « accueillir » les primo-arrivants dont il permet l'enfermement jusqu'à 25 jours avant leur libération ou leur transfert dans une autre structure fermée. Surveillé par la police nationale, il est placé sous la direction d'un "service de premier accueil 24 »composé de fonctionnaires du ministère de la protection du citoyen et prévoit l'intervention d'un service d'asile, d'agents du HCR (Haut commissariat des Nations unies pour les réfugiés), d'interprètes, d'une équipe médicale de Médecins du monde et d'agents de nettoyage.

Contrairement à ce que son statut laisse présumer, l'installation du centre ne signe pas la fin des pratiques arbitraires mais tend plutôt à les perpétuer sous d'autres modalités. D'une part, le dispositif est placé dès son ouverture sous le signe de l'urgence. Face à de nouvelles arrivées de migrants à l'été 2012 (figure 2), le ministère de la protection du citoyen décide de créer dans un premier temps une " unité mobile ", centre temporaire qui peut être installé et supprimé en fonction des flux migratoires. Le statut du dispositif, destiné à devenir un KEPY sans l'être pour autant, est si incertain que ses employés eux-mêmes s'y perdent : «On a du mal à comprendre ce que la loi exige et quelles sont les procédures à suivre. (...) Les agents ne savent pas exactement quel rôle ils ont. Il y a certes la loi, mais aussi beaucoup de questions d'interprétation 25 », résume la directrice du centre. Cette gestion entrâne une série

22. La loi 3907/2011 - "Establishment of an Asylum Service and a First Reception Service" - est disponible en anglais sur le site du ministère de la protection du citoyen [http://www.mopocp.gov.gr/index.php?option=ozo_content\&perform=view\&id=3518\&Ite mid $=466 \&$ lang $=\mathrm{EN}]$.

23. Directive 2008/115/CE du Parlement européen et du Conseil du 16 décembre 2008 relative aux normes et procédures communes applicables dans les États membres au retour des ressortissants de pays tiers en séjour irrégulier (dite "directive retour »). Cette directive comporte 23 articles consacrés à la fin du séjour irrégulier (décisions de retour, départs volontaires, éloignements, interdictions d'entrée), aux garanties procédurales (notifications des décisions, voies de recours, assistance juridique en attente du renvoi), à la rétention (motifs, conditions et limites de l'enfermement) et aux modalités de transposition de la directive.

24. Service mis en place par la loi 3907/2011. 
de problèmes organisationnels et matériels (service de demande d'asile indisponible, chauffage insuffisant, fuites d'eau, manque de vêtements) qui privent les étrangers enfermés de certains droits fondamentaux. D'autre part, au-delà de ces problèmes circonstanciels, l'installation du centre tend à institutionnaliser une forme d'exception. Sous couvert d'accueil, la loi non seulement autorise mais finalement entérine et normalise la détention systématique des primo-arrivants, quel que soit leur statut (mineurs, demandeurs d'asile, personnes vulnérables, etc.) dans des locaux qui, faute d'être définis, peuvent être organisés sur un mode carcéral et réduits au strict nécessaire.

\section{Ports et commissariats : des espaces en marge mais structurants}

En dehors des espaces consacrés, certains lieux de l'île sont détournés de leur fonction pour servir à la rétention des étrangers : les commissariats et les ports, y compris les plus petits, employés chroniquement depuis plus d'une décennie. Si leur utilisation, ponctuelle et irrégulière, peut sembler secondaire, ils jouent en fait un rôle déterminant dans le contrôle migratoire à l'échelle locale, assurant sa continuité dans le temps et dans l'espace. Non seulement ils font office de lieux de confinement en l'absence d'infrastructures prévues à cet effet, mais ils servent aussi de passerelles entre les différents dispositifs policiers en dehors de ces périodes.

Le commissariat de Mytilène en est l'exemple type. Premier lieu d'enfermement des étrangers à la fin des années 1990 et seul espace de détention officiel de l'île, il est régulièrement mobilisé depuis pour pallier l'absence de centre de rétention. Son utilisation fait l'objet d'un consensus parmi les acteurs du contrôle mais les conditions d'enfermement y sont particulièrement mauvaises (cellules sombres et collectives, locaux vétustes, surpopulation fréquente, etc.) ${ }^{26}$ et seul un nombre limité de migrants peut y être enfermé. La reprise soudaine des arrivées au mois d'août 2012 a montré les limites de cette solution provisoire employée depuis une décennie. Interrogé quelques semaines avant, un responsable du commissariat déclarait : "On n'a pas de plan. Si les migrants viennent on n'a pas de centre. (...) On attend les ordres d'Athènes 27 ». L'absence de prévision des arrivées, pourtant chroniques depuis les années 2000 , a en effet abouti à une situation chaotique dans les mois suivants. Entre août 2012 et septembre 2013, des étrangers sont successivement enfermés au commissariat, dans le port ou laissés à la rue, les gardecôtes et la police se renvoyant la responsabilité de leur prise en charge. Des migrants en viennent même à camper devant le commissariat afin d'être interpellés, seul moyen pour eux d'obtenir une obligation à quitter le territoire

25. Entretien avec la responsable du centre de Moria, fonctionnaire du service de premier accueil, décembre 2013, Mytilène, LT.

26. Notes de terrain, novembre 2013, Mytilène, LT.

27. Entretien avec le responsable du département de la sécurité à Mytilène, août 2012, Mytilène, LP. 
grec ${ }^{28}$ qui leur permet de prendre le ferry ; mais les autorités, faute de directives claires et de financements à cet effet, refusent de s'en occuper. Si les commissariats sont moins sollicités avec les ouvertures successives de l'ancienne prison, de Pagani puis de Moria, ils ne disparaissent pas pour autant du paysage du contrôle migratoire. Utilisés en amont, en aval ou parallèlement à ces derniers, ils n'ont jamais cessé de servir d'antichambres aux centres de rétention, alternant plusieurs fonctions : détenir temporairement les étrangers avant leur transfert dans les centres, notamment dans les villes portuaires de l'île (Molyvos, Kalloni), compenser l'absence de place en cas de surpopulation des dispositifs et emprisonner les individus soupçonnés d'être des passeurs dans l'attente de leur procès.

Fonctionnant en miroir avec les commissariats, les ports jouent un rôle proche sans s'y réduire complètement, les étrangers interceptés en mer étant sous la responsabilité des garde-côtes et donc conduits dans le port de Mytilène. À la détention archétypale du commissariat - des cellules vétustes partagées avec des détenus de droit commun - s'oppose au port un espace extérieur, à l'air libre, destiné à la circulation des voyageurs. Lieu de passage pour les uns et de blocage pour les autres, le port matérialise les ambivalences de la frontière comme zone de contact et de séparation, accueillant tout à la fois des bateaux de plaisance, des ferries venant de Turquie et des embarcations de fortune utilisées par les migrants. Dans cet espace de transit à deux vitesses, la durée d'enfermement dépend de la présence ou non d'un centre de rétention sur l'île. Entre août 2012 et septembre 2013 notamment, l'absence d'infrastructure et le manque de places au commissariat ont conduit les gardecôtes à détenir des migrants pendant plusieurs semaines alors qu'aucun financement ni dispositif n'étaient prévus pour les prendre en charge, hormis quelques tentes et matelas en mousse. Doublement « enfermés dehors 29 » - de l'autre côté de la frontière (dans la zone internationale) et à ciel ouvert -, les personnes étaient détenues entre des carcasses de voiture confisquées par les autorités et le quai des ferries, exposées derrière une grille à la vue de tous.

En prolongeant, complétant et précédant le maintien dans les dispositifs officiels, le port et les commissariats forment donc une sorte d'extension des centres au-delà de leurs barrières, offrant des espaces d'enfermement flexibles, aisés à mobiliser dans l'urgence mais soustraits aux obligations matérielles et procédurales que les dispositifs officiels sont censés, au moins selon la législation, assurer.

28. Ce document enjoint les étrangers à quitter le territoire grec dans un délai d'un mois ( $c f$. article 22 de la loi 3907/2011). A partir de 2012, cette durée à été exceptionnellement étendue à six mois renouvelables pour les Syriens. Le document, qui comporte une photo d'identité et certaines informations personnelles, constitue par défaut une autorisation de séjour en Grèce pendant cette période, excluant certaines régions telles que l'Achaïe et la Thesprotie, Athènes et Corfu.

29. Notion relevée par Michel Agier dans un texte prononcé par Michel Foucault au cours duquel il évoque les boat people comme étant des «enfermés dehors » : Agier M., «Quels 
L'intérêt d'un cas comme Lesbos est aussi qu'il conduit à décentrer le regard au-delà des dispositifs officiels pour s'intéresser à leurs marges, souvent peu visibles mais non moins importantes. En l'absence de procédure claire, les migrants sont chroniquement enfermés dans des lieux privés transformés pour l'occasion - restaurant, bateau ou salle polyvalente - qui n'ont en commun que leur altérité. Les autorités y ont recours car, faute de moyens affectés au transport des migrants interceptés sur l'île, elles sont contraintes d'utiliser leurs fourgons (pas toujours disponibles ou en nombre insuffisant) ou de réquisitionner des bus municipaux et privés, ce qui peut prendre du temps.

Les locaux utilisés en attendant le transfert des migrants vers les lieux d'enfermement officiels n'assurent aucune garantie juridique ni matérielle, l'accès aux soins, à l'eau et à la nourriture étant conditionné à l'endroit choisi et à la disponibilité des habitants. Le caractère informel des lieux rend par ailleurs difficile le contrôle des pratiques policières par des acteurs associatifs, juridiques ou médicaux, lesquels sont rarement prévenus des arrivées et peuvent difficilement intervenir au vu des distances à parcourir. Soustraits au regard public et déconnectés de la hiérarchie policière, ces lieux sont en fait soumis à une gestion empirique guidée moins par les procédures que par les « imprévus de la situation » et les « routines professionnelles 30 » des policiers. Cette situation ouvre la voie à des pratiques discrétionnaires justifiées au nom de l'urgence et d'un certain pragmatisme - l'idée qu'il faut « faire avec les moyens du bord 31 » revient comme un leitmotiv parmi les policiers rencontrés. Si ces locaux se caractérisent par leur précarité, leur disparité et la brièveté de leur utilisation, ils ont scandé l'histoire de la rétention sur l'île depuis les premières arrivées et continuent à rythmer son quotidien. Non seulement les interstices du contrôle n'ont pas disparu avec l'institutionnalisation du dispositif mais ils constituent même, d'un point de vue socio-historique, une des rares permanences d'un système - si tant est qu'on puisse l'appeler ainsi marqué avant tout par son instabilité.

S’intéresser aux marges du dispositif revient enfin à se défaire du prisme de l'enfermement pour prêter attention à ce qui se passe en amont et en aval de la privation de liberté. À Lesbos, il n'est pas rare que les policiers refusent de se déplacer alors que des habitants leur indiquent la présence de migrants sur l'île, ces derniers étant alors contraints de marcher pendant des dizaines de kilomètres pour rejoindre la capitale où, à certaines périodes, ils doivent encore faire plusieurs allers-retours dans la ville avant d'être pris en charge

temps aujourd'hui en ces lieux incertains ? ", L'Homme, 185-186, 2008, pp. 105-120 ; Kobelinsky C., Makaremi C. (eds.), Enfermés dehors. Enquêtes sur le confinement des étrangers, Bellecombe-en-Bauges, Éditions du Croquant, 2009, p. 335.

30. Fischer N., op. cit.

31. Entretien avec un policier, août 2013, Molyvos, LT. 
(figure 3). Les distances parcourues dessinent ainsi les contours d'une sorte d'enfermement dans la circulation où l'île elle-même devient comme une prison. Ce phénomène s'observe également à l'échelle du quartier comme à l'automne 2012 où des migrants se sont réfugiés dans un parc pendant plusieurs nuits après avoir été ignorés par chaque autorité. $\mathrm{Si}$, à proprement parler, ils n'étaient pas enfermés dans cet espace public par excellence, ils n'avaient guère d'autre choix que de s'y trouver, pris au piège d'une île dont il est quasiment impossible de s'échapper en l'absence de document de voyage valide. Cette situation montre que le caractère contraignant de la privation de liberté ne réside pas toujours dans l'entrave à la mobilité mais peut au contraire «se nicher dans le déplacement et le contrôle de celle-ci 32 » : les étrangers se trouvent assignés à des lieux ouverts et à des circulations qui, paradoxalement, établissent et prolongent l'enfermement.

Figure 3 Enfermez-moi !

Parcours de migrants sur l'île de Lesbos en octobre 2013

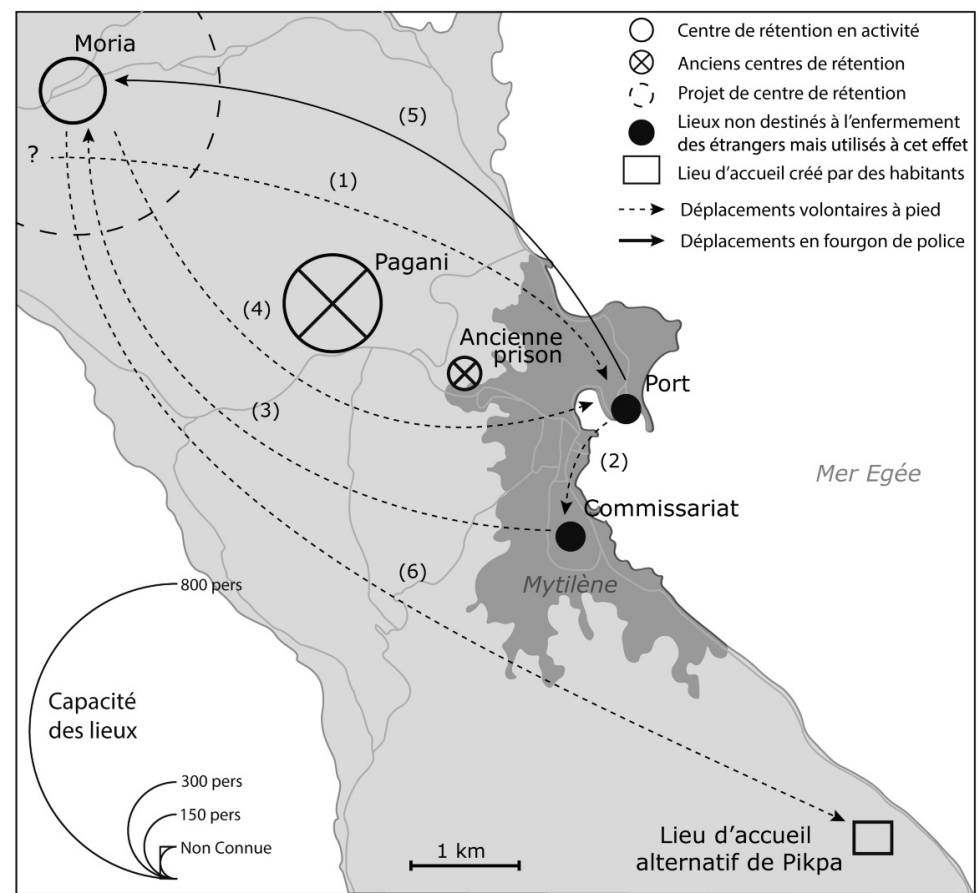

Hamid, Nassim et Aziz ont été rencontrés sur les quais de Mytilène en octobre 2013. Arrivés quelques jours plus tôt à une extrémité de lîle avec une dizaine de personnes, ils cherchaient en vain à se faire arrêter. Après s'être adressés aux garde-côtes sur les conseils d'un passant (1), ils sont dirigés vers le commissariat situé à $2 \mathrm{~km}$ de là (2) mais les policiers refusent de les recevoir et les renvoient vers le centre de Moria. Après une marche de $8 \mathrm{~km}$ environ (3), le responsable du centre leur explique que les policiers ne sont pas habilités à arrêter des individus dans la zone et leur indique la direction du port (4) où nous les rencontrerons quelques heures plus tard, épuisés et déconcertés. Ils seront finalement arrêtés sous la pression d'acteurs associatifs et conduits au centre de Moria (5) d'où, une fois relâchés, ils se rendront à Pikpa (6). 
L'indifférence des autorités, qu'on pourrait presque qualifier d'institutionnalisée tant elle a structuré le rapport aux étrangers pendant plus d'un an, est d'autant plus surprenante que le gouvernement grec lançait à la même période l'opération «Xenios Zeus » au cours de laquelle des milliers d'étrangers en situation irrégulière ont été arrêtés et enfermés ${ }^{33}$. Cette contradiction s'éclaire néanmoins à l'aune de l'histoire de l'île que nous avons tenté d'esquisser : si les autorités nationales et locales ont géré les arrivées de migrants par à-coups successifs pendant une quinzaine d'années, c'est sans doute que Lesbos constitue à plus d'un titre (ou tout au moins a constitué jusqu'aux années 2010), pour détourner l'expression de Dominique Schnapper ${ }^{34}$, une île de migrations qui s'ignore, refusant de considérer les flux migratoires comme une réalité amenée à durer.

La diversité des lieux créés ou utilisés à des fins d'enfermement administratif à Lesbos soulève finalement plusieurs enjeux. Premièrement, la genèse de la rétention sur l'île montre à quel point la gestion des flux migratoires, qui procède par tâtonnements au fil des arrivées, s'inscrit dans la socio-histoire du camp. D'une part, les lieux de rétention à Lesbos se situent au croisement d'une logique répressive visant à séparer les individus et d'une logique humanitaire d'assistance aux primo-arrivants, ambivalence caractéristique des camps coloniaux et contemporains décrits par Marc Bernardot 35 . Cette tension, si elle est intrinsèque au statut de la rétention, se manifeste avec acuité sur l'île où les dispositifs ont toujours oscillé entre accueil et mise à l'écart : une prison ouverte que l'on referme, un entrepôt symbole de l'exception devenu lieu de passage, un KEPY où l'on « accueille » entre les grilles. D'autre part, en dépit de leur disparité, les lieux de rétention de l'île ont en commun leur plasticité, c'est-à-dire leur capacité à être transformés en fonction des situations. Fondés sur des locaux précaires - sites désaffectés, détournés de leur fonction ou faciles à construire et démonter (préfabriqués) -, ils se caractérisent par une certaine souplesse qui paradoxalement garantit leur stabilité. Or c'est là l'enjeu de la définition du camp : «l'exception se réalise » à travers lui comme un « état permanent » qui s'incarne dans un « lieu concret 36 » ou plutôt, ici, des lieux concrets. Cependant, à Lesbos, l'officialisation progressive de la rétention ne fait pas disparaitre l'arbitraire mais tend à l'institutionnaliser : en légitimant le recours à l'enfermement comme mode de gestion des étrangers, elle normalise une situation qui n’a guère changé par rapport à la décennie précédente.

32. Darley M. et al., op.cit., p. 15.

33. Lancée le 5 août 2012, l'opération Xenios Zeus visait à augmenter les arrestations d'étrangers en situation irrégulière à Athènes. De nombreuses craintes de détention et d'expulsions arbitraires ont été exprimées par les organisations de défense des droits humains. Cf. Frontex entre Grèce et Turquie, la frontière du déni, rapport FIDH - Migreurop - REMDH, mai 2014, pp. 75-76.

34. Schnapper D., La France de l'intégration, sociologie de la nation en 1990, Paris, Gallimard, 1991, p. 13.

35. Bernardot M., Camps d'étrangers, Bellecombe-en-Bauge, Éditions du Croquant, 2008, p. 223.

36. Fischer N., op. cit. 
Deuxièmement, elle souligne que les « manifestations punctiformes 37 » de la frontière territoriale dont les centres de rétention font partie sont nombreuses. Au-delà des murs des structures officielles, d'autres frontières marquent le seuil d'un " régime de confinement 38 » hétéroclite qui révèle la démultiplication contemporaine des formes de contrôle. Aussi, le cas de Lesbos invite à penser la rétention moins comme un lieu fermé que comme une chaîne de dispositifs et de pratiques policières, intimement liés les uns aux autres, qui servent non seulement à immobiliser les personnes mais aussi à orienter leurs déplacements. Il entre ainsi en résonance avec le paysage du contrôle migratoire dans l'UE où, du campement de Patras au detention centre britannique, du centre d'accueil de Lampedusa au commissariat parisien, les étrangers sans papiers font l'expérience d'une «vie encampée 39 » qui les enferme tour à tour à l'intérieur et à l'extérieur, entre des grilles, dans des bidonvilles et dans la circulation, selon des dynamiques moins contradictoires que complémentaires qui façonnent les parcours migratoires.

Le cas de Lesbos vient ainsi interroger le lien entre relégation et insularité. À l'instar du rôle joué historiquement par les îles dans le contrôle des mobilités ${ }^{40}$, Lesbos constitue à la fois un espace de confinement, les migrants n'ayant quasiment aucune chance de la quitter sans l'intervention de la police, et un territoire de confins, son caractère insulaire contribuant, comme dans d'autres îles de la Méditerranée, à la mise en scène des frontières extérieures de l'UE et à la « mise en visibilité de l'urgence humanitaire associée à la migration 41 ». En témoignent en particulier les arrivées de l'été 2015 qui, relayées jusqu'aux États-Unis, ont été présentées comme emblématiques du "problème » de l'immigration clandestine en Europe ${ }^{42}$.

Cependant, l'étude des lieux de rétention sur l'île montre que l'enfermement peut justement, dans certains cas, nuancer l'effet de « barrière insulaire 43 », c'est-à-dire estomper, voire briser, l'enclavement produit a priori par l'insularité. À Lesbos, l'arrestation des étrangers et leur placement en rétention contribuent en effet le plus souvent à faire circuler les individus, qui sont soit envoyés dans d'autres centres de rétention du pays (auquel cas il s'agit d'une mobilité forcée dessinant des « itinérances rétentionnaires 44 »), soit libé-

37. Cuttitta P., "Un monde frontière. Le contrôle de l'immigration dans l'espace globalisé », Cultures E Conflits, 68, 2007, pp. 61-84 ; Clochard O., "Le contrôle des flux migratoires aux frontières de l'Union européenne s'oriente vers une disposition de plus en plus réticulaire », Carnets de géographes, 2010.

38. Akoka K., Clochard O., op. cit.

39. Agier M. et Lecadet C., Un monde de camps, Paris, La Découverte, 2014, p. 422.

40. Briskman L., Mountz A., "Introducting island: the placement of asylum seekers and migrants on islands", Shima: the international journal of research into island cultures, 6/2, pp. 21-26.

41. Bernardie-Tahir N., Schmoll C., "Iles, frontières et migrations méditerranéennes : Lampedusa et les autres », L'Espace politique, 1/25, mis en ligne le 5 avril 2015 [http://espacepolitique.revues.org/3381].

42. New York Times, “On Island of Lesbos, a microcosm of Greece’s other crisis: migrants”, 4 août 2015.

43. Akoka K., Clochard O., op. cit. 
rés avec une obligation à quitter le territoire ou une carte de demandeur d'asile qui leur permet d'acheter un billet de transport (auquel cas il s'agit d'une mobilité concédée et limitée dans le temps). Il faut en effet savoir que, sous l'effet d'injonctions européennes aux transporteurs privés ${ }^{45}$, les entreprises délivrant les tickets de ferry sont tenues de vérifier le statut des étrangers. Le passage par le système de contrôle, en permettant d'obtenir une autorisation de séjour par défaut, offre donc un précieux sésame pour la suite du parcours migratoire. Non seulement il n'est plus synonyme d'immobilisation - ou alors temporaire -, mais c'est une condition nécessaire à la poursuite du voyage au point que des migrants, soutenus par des militants, cherchent à se faire arrêter. À certaines périodes, la mobilité des étrangers a même été prise en charge et organisée par les autorités, qui ont acheté des billets de ferries (en 2008) ou affrété des navires depuis Athènes (en 2015) afin de désengorger les îles. Dans ce contexte, être enfermé plus longtemps a parfois permis, paradoxalement, de quitter plus rapidement l'île.

«Les migrants qui n'avaient pas d'argent avaient la possibilité de partir grâce à la préfecture mais seulement les jours ou la compagnie faisait les allers-retours, c'est-à-dire le mardi, le jeudi et le dimanche. Il fallait donc par moment lancer une procédure pour demander au préfet et à la police de garder quelques jours de plus les gens qui étaient susceptibles de prendre le bateau 46 ».

Le cas de Lesbos souligne ainsi la nécessité de ne pas surévaluer la dimension enclavante de l'insularité dans l'étude des îles situées aux frontières de l'UE, le régime de confinement fonctionnant en partie comme un « moteur de circulation et de redistribution des flux 47 ». Si l'enfermement dans ces espaces éloignés peut contribuer à tenir physiquement et symboliquement les migrants à l'écart, comme c'est le cas par périodes à Malte, à Chypre, à Lampedusa 48 et à Lesbos ${ }^{49}$, il peut aussi conduire à les faire partir au plus vite, que les autorités souhaitent attirer l'attention sur leur gestion du phénomène

44. Michalon B., «La mobilité au service du mouvement », Géographie et cultures, 81, 2012, pp. 91-110.

45. Guiraudon V., «Logiques et pratiques de l'État délégateur : les compagnies de transports dans le contrôle migratoire à distance », Cultures $E$ Conflits, 45, 2001, pp. 51-63.

46. Entretien avec une ancienne employée de Pagani, février 2010, Mytilène, LP.

47. Akoka K., Clochard O., op. cit.

48. Mountz A., "The enforcement archipelago: detention, haunting and asylum on islands", Political Geography, 30/3, 2011, pp. 118-128 ; Lemaire L., "Islands and a Carceral environment: maltese policy in terms of irregular migration", Journal of immigrant and refugee studies, 12/2, 2014, pp. 143-160 ; Akoka K., Clochard O., op. cit. ; Tassin L., "Accueillir les indésirables. Les habitants de Lampedusa à l'épreuve de l'enfermement des étrangers », Genèses, 3/96, pp. 110-131.

49. En particulier durant les premières années de Pagani où des migrants étaient détenus à l'abri des regards pendant des mois à Lesbos. De même, l'installation d'un « centre de pré-expulsion » sur l'île amorcée en 2014, qui permet d'enfermer jusqu'à 18 mois les étrangers en situation irrégulière en vue de leur éloignement, renoue avec le rôle historique des îles dans le contrôle à distance des populations indésirables. 
ou, au contraire, soustraire les étrangers aux regards et aux projecteurs médiatiques. Le cas de Lesbos invite ainsi à ne pas réduire les îles à l'isolement qui les caractérise a priori mais à prêter attention aux différents « usages » 50 qui en sont faits et à se demander si, comme pour les camps évoqués plus haut, ce ne serait pas justement la plasticité des îles, tour à tour lieux de mise en scène ou d'invisibilisation des questions migratoires, qui font d'elles un enjeu des frontières européennes.

\section{Du blindage des frontières à celui des habitants ? La société civile à l'épreuve de l'enfermement}

Si, comme nous venons de le voir, les lieux de rétention se caractérisent à Lesbos par une certaine opacité, ils ne sont pas pour autant hermétiques à la critique. Au contraire, les mauvaises conditions d'enfermement et l'absence chronique de prise en charge officielle des migrants ont provoqué des réactions à plusieurs échelles, des habitants de l'île au Conseil de l'Europe en passant par des militants internationaux. Le régime d'exception a constitué en fait un moteur des mobilisations, suscitant des engagements individuels dans les lieux d'enfermement, des actions collectives protestataires à l'extérieur et des alternatives à la détention relativement inédites dans l'Union européenne ; mais ces différentes initiatives ont aussi contribué plus ou moins directement, et non sans ambivalences, à façonner les pratiques du contrôle.

\section{Se mobiliser (ou pas) contre la rétention : esquisse des ressorts de la contesta- tion}

L'observation du dispositif au cours et en dehors des périodes de médiatisation permet d'analyser les conditions d'émergence des mobilisations dirigées contre la rétention, qu'elles soient motivées par le refus de l'enfermement des migrants ou, à l'inverse, le rejet de ces derniers. Si le recours à la rétention fait l'objet d'un consensus général sur l'île, trois facteurs semblent déterminants dans son acceptation ou, au contraire, sa contestation : la visibilité ou non des dispositifs, les éventuelles retombées économiques sur la société locale et les dispositions des acteurs concernés, tant dans le champ militant que politique.

Le premier enjeu est celui de la visibilité des étrangers. Si cette dernière a pu susciter des réactions d'inquiétude voire d'aversion à l'égard des migrants, comme dans le cas de l'ancienne prison où des plaintes de voisins ont abouti à sa fermeture, elle a été aussi un facteur de mobilisation en leur faveur. Alors que l'enfermement dans les cellules du commissariat n'a jamais suscité de réactions en dehors des professionnels habilités à y accéder, le maintien des migrants dans le port aux yeux de tous à partir de l'été 2012 a provoqué des 
réactions sans précédent sur l'île, fédérant des soutiens bien au-delà des réseaux militants et associatifs. Il faut dire que les étrangers se trouvaient non seulement dans la zone de transit internationale mais aussi à l'extrémité du port de plaisance, lieu de promenade par excellence des touristes et des habitants. Cependant, c'est aussi l'absence de visibilité qui peut à l'inverse entrâ̂ner des protestations, comme dans le cas de Pagani où l'opacité du centre et les difficultés rencontrées pour y accéder ont conduit militants locaux et internationaux à se rassembler. La visibilité n'était alors plus seulement un motif de la contestation mais tout à la fois son levier (être $v u$ des autorités avec des manifestations aux abords du centre) et son but (réussir, en s'approchant, à voir le centre et des retenus). Les rassemblements ont été d'ailleurs d'autant plus efficaces que leur médiatisation a offert une visibilité internationale au lieu via des réseaux de militants et de journalistes qui ont donné à voir, au sens propre comme au sens large, par des images et par des textes, les conditions de rétention. Pagani l’inaccessible est devenu Pagani le symbole, la visibilité jouant ainsi un rôle performatif au point que les photographies de cette époque étaient encore utilisées en 2015 pour illustrer des articles sur l'enfermement des étrangers en Grèce ${ }^{51}$.

Un second enjeu des mobilisations réside dans les intérêts économiques que peuvent ou non y trouver les acteurs de l'île. Sur un territoire où le PIB est environ de $20 \%$ inférieur à l'échelle nationale ${ }^{52}$, dans un contexte de crise accentuée depuis la fin des années 2000 , les lieux de rétention représentent une ressource non négligeable qui participe à leur acceptation dans la société locale. À Pagani par exemple, la location de l'entrepôt pendant cinq ans sans restructuration du bâtiment a profité aux entrepreneurs de l'île : non seulement la restauration, la manutention ou encore l'évacuation des eaux usées ont été confiées sur appel d'offre à des entreprises pour l'année, mais toute une série de fournitures (vêtements, nécessaire d'hygiène, médicaments) ont été achetées de façon ponctuelle auprès de petits commerçants choisis à tour de rôle pour répartir équitablement les bénéfices induits par ce nouveau marché ${ }^{53}$. Parallèlement, l'absence d'intérêts a pu susciter des protestations contre la rétention. Pour la construction du centre de Moria par exemple, le ministère a opté pour une procédure d'urgence lui permettant de limiter légalement le marché à une liste de grandes entreprises spécialisées, ce qui excluait de fait les entrepreneurs de l'île qui se sont mobilisés, en vain, afin d'obtenir la possi-

51. Les Inrocks, «Grèce, l'avenir de milliers d'immigrés détenus se joue aussi lors des législatives », 21 janvier 2015 [http://www.lesinrocks.com/2015/01/24/actualite/grece-lavenir-demilliers-dimmigres-detenus-se-jouent-aussi-lors-des-legislatives-11550036/]; Worldbulletin, "Greece pledges to shut immigrant detention centres", 14 février 2015 [http://www.worldbulletin.net/greece/155048/greece-pledges-to-shut-immigrantdetention-centres].

52. Eurostat [t_nama_reg] 2005 à 2011.

53. Archives de la préfecture de Lesbos consultées en mars 2010 et en août 2012 ; archives du ministère de l'Intérieur consultées à Athènes en mai 2011, LP. Selon ces sources, les produits d'hygiène et médicamenteux fournis par la préfecture aux personnes enfermées à Pagani ont représenté environ 300000 euros de dépense entre 2005 et 2009. 
bilité d'y candidater ${ }^{54}$. De la même manière, les habitants de la commune de Moria rassemblés en réunions collectives quelques semaines après l'inauguration du centre s'inquiétaient de son ouverture et regrettaient de ne pas avoir été informés ni associés d'une manière ou d'une autre, sur le plan économique, au projet 55 .

Enfin, un dernier facteur déterminant des mobilisations a été la disponibilité ou non de forces militantes et partisanes à même de problématiser dans l'espace public la question de la rétention. Il a fallu, d'abord, un minimum de forces vives au sein de la société locale, ce qui n’a pas toujours été évident. Comme l'explique un membre de Prosfigui, premier collectif de soutien aux réfugiés créé au milieu des années 2000 , la rétention faisait partie du paysage militant sans en être une priorité, les quelques actions menées en faveur des migrants étant principalement guidées par des objectifs humanitaires ou religieux, ce qui a décidé ses membres à « porter un premier discours politique ${ }^{56}$ » sur le sujet. La convergence entre différents acteurs et plusieurs échelles de mobilisation semble alors avoir été décisive. Si les militants de Prosfigui tentaient de mobiliser les réseaux associatifs et militants de l'île, c'est la présentation à Bruxelles d'un rapport de l'organisation allemande Pro Asyl ${ }^{57}$ qui, répercutée dans la presse et les télévisions locales, a réellement donné de l'ampleur à la protestation. Renforcé par d'autres rapports d'ONG et même du CPT ${ }^{58}$, cet élan a conduit à l'organisation d'un camp No Border sur l'île en août 2009 et abouti à la fermeture définitive du centre en octobre de la même année. Malgré de vives tensions entre militants et autorités durant l'été, au point que des renforts exceptionnels ont été dépêchés sur l'île, les actions menées à cette occasion ont permis, de l'aveu même de la police et du préfet, de faire connaître la situation au-delà de l'île : « [NoBorder] a été positif parce que [les militants] ont montré à beaucoup de gens la situation, et c'est peutêtre pour cela que le ministre est venu visiter Pagani. On manquait de tout : pas assez d'officiers, pas assez de docteurs et pas assez d'argent pour chauffer l'entrepôt pendant l'hiver ${ }^{59}$ ».

Ce jeu d'échelles à géométrie variable se retrouve à travers les périodes, les manifestations contre Moria et l'organisation d'un camp d'accueil alternatif appelé PIKPA étant, pour ne citer qu'eux, soutenus par le collectif d'informations aux réfugiés Welcome2Europe, lui-même constitué en partie d'anciens participants au No Border. La convergence d'intérêts ne doit cependant pas

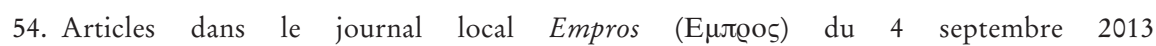
[http://www.emprosnet.gr/article/49407-apokleismos-proklisi] et du 19 septembre 2013 [http://www.emprosnet.gr/article/49817-horoi-kratisis-monimoi].

55. Entretiens avec une habitante du village de Moria et une militante active sur l'île, novembre 2013, Mytilène, LT.

56. Entretien avec un ancien membre de Prosfigui, décembre 2013, Mytilène, LT.

57. Rapport de Pro Asyl, "The trust must be bitter", octobre 2007.

58. Cf. note 20.

59. Entretien avec un officier de police, février 2012, Mytilène, LP. 
masquer les difficultés d'organisation et les conflits induits par le rapprochement d'acteurs issus d'horizons différents, en particulier pendant et après le camp No Border dont les effets ont été ambivalents, les étrangers se trouvant par la suite enfermés dans le port sans aucune infrastructure et une prise en charge minimale. Ce sont enfin les acteurs politiques qui peuvent servir de relais ou de moteur aux protestations, tel le maire de Lesbos qui, en octobre 2013, organisait la visite d'une délégation du conseil municipal de l'île au centre de Moria et dénonçait les conditions de rétention pour prôner un « véritable accueil digne de ce nom 60 », légitimant ainsi les revendications portées jusque-là par quelques voix marginalisées. À la même période, le parti néonazi «Aube dorée » organisait en opposition des rassemblements dans le village jouxtant le centre, critiquant les dépenses de l'État en faveur des étrangers au détriment de la population locale.

Visibilité, intérêts économiques et vivier militant : ces différents facteurs, présentés ici séparément dans un souci de clarté, se combinent dans la pratique et c'est uniquement de leur imbrication que peuvent surgir les protestations. Le processus de mobilisation semble au fond guidé par une logique de seuil qui, une fois dépassé, produit des situations de rupture qui contraignent les autorités à agir : transformer la prison en lieu de privation de liberté, l'abandonner, ouvrir Pagani, fermer Pagani, construire Moria, toutes ces étapes sont intimement liées aux réactions de la société locale qui, progressivement, ont modelé les pratiques du contrôle. En contestant le dispositif, ses opposants ont malgré eux contribué à sa normalisation. En dénonçant publiquement les conditions de rétention à Pagani et en revendiquant la prise en charge des migrants par les autorités lorsque ces derniers étaient laissés à eux-mêmes dans la rue ou enfermés dans le port, les acteurs mobilisés en faveur des migrants ont contraint les autorités à agir. Or l'institutionnalisation de la rétention a, comme en France 61, déplacé le débat sur la légitimité ou non de l'internement vers celui, plus consensuel, des modalités du placement. Après une année 2012 chaotique en l'absence d'infrastructures, les autorités locales et toute une partie du champ associatif et des ONG attendaient avec impatience l'ouverture $\mathrm{du}$ « centre de premier accueil » de Moria, lieu de détention perçu comme relativement moderne, souple et adapté. Cette évolution a divisé le milieu contestataire entre opposants à la rétention et partisans d'une amélioration du dispositif de contrôle, dont certains ont choisi de travailler dans les lieux d'enfermement mais se sont vite confrontés aux limites de cette activité.

60. Entretien avec le maire de Lesbos, août 2013, Mytilène, LT ; article du 3 septembre 2013 paru au journal Empros [http://www.emprosnet.gr/article/49185-moriana-organa].

61. Fischer N., «La rétention administrative dans l'État de droit. Genèse et pratique du contrôle de l'enfermement des étrangers en instance d'éloignement du territoire dans la France contemporaine », thèse de science politique, IEP de Paris, 2007. 
Dans une société insulaire comme l'île de Lesbos, les professionnels du secteur de la migration n'ont pas beaucoup d'opportunités d'emploi en dehors des dispositifs fermés. Durant les cinq années que couvre la période de recherche de cet article, nous avons pu observer que de mêmes acteurs se retrouvent tour à tour au sein des différentes structures. Infirmière, employés de Médecins sans frontières ou de Médecins du monde, du HCR ou encore de l'OIM (Organisation internationale pour les migrations) interviennent successivement dans l'ancienne prison, à Pagani, à Moria et dans les espaces alternatifs, passant parfois d'une organisation à l'autre. De même, plusieurs migrants arrivés à Lesbos dans les années 2000 sont devenus interprètes dans les lieux d'enfermement, travaillant pour l'État et des ONG ou comme bénévoles dans des milieux contestataires. Enfin, plusieurs militants ont participé à des programmes sociaux mis en place au sein des institutions fermées et dans des structures ouvertes, ce qui pose la question de la professionnalisation du militantisme et des marges d'actions possibles dans ce contexte ${ }^{62}$.

Plusieurs facteurs conduisent à faire l'hypothèse que le travail en rétention exerce à Lesbos un certain effet «blindant » sur les employés chargés de l'assistance sociale, juridique et médicale aux personnes enfermées. Quel que soit le degré d'engagement des intervenants, il semble en effet que leur tolérance aux situations de violence et de détresse s'élève au fur et à mesure que leur expérience professionnelle s'épaissit, comme si la confrontation quotidienne aux conditions de rétention contribuait à normaliser ces dernières. « Après seulement quelques semaines, $\mathrm{j}$ 'acceptais des choses que je n'aurais jamais pensé pouvoir supporter $63 »$ résume un ancien travailleur social de Pagani. Cette accoutumance, interprétée par certains intervenants comme un «mécanisme de défense » face à la violence des lieux ${ }^{64}$, pose problème en matière de respect des droits des personnes, lesquels risquent alors d'être appréhendés à l'aune d'une échelle de valeurs subjective et relative au site observé. Plusieurs intervenants engagés dans la défense des étrangers racontent ainsi comment, alors qu'ils se pensaient particulièrement attentifs aux traitements dégradants, donc préservés de leur banalisation, leur sensibilité s'est peu à peu transformée sous l'effet conjoint de l'habitude et des objectifs professionnels, comme en témoigne ce militant actif dans les luttes de soutien aux migrants avant d'être employé à Pagani.

62. Sur les ambiguïtés de l'intervention des associations de défense des étrangers dans les institutions étatiques, voir : Fischer N., « Jeu de regards. Surveillance disciplinaire et contrôle associatif dans les centres de rétention administrative », Genèses, 75/2, 2009, pp. 45-65 ; Pette M., "Associations : les nouveaux guichets de l'immigration ? Du travail militant en préfecture ", Sociologie, 5/4, 2014, pp. 405-421.

63. Entretien avec un ancien employé de Pagani, novembre 2013, Mytilène, LT.

64. Entretien avec un ancien employé de Pagani, novembre 2013, Mytilène, LT. 


\begin{abstract}
«Après deux, trois, quatre mois, j’ai remarqué que je regardais [les étrangers] comme des outils. Ce que je veux dire par là, c'est que je ne prêtais plus attention à leur personne et à leur histoire, leur histoire personnelle. Je prêtais attention à leur histoire personnelle oui, mais parce que c'était très important : ça nous donnait des moyens de pression sur les autorités, ça permettait de changer quelque chose dans le système. Donc je ne les regardais pas comme des êtres humains mais comme un instrument pour mon travail, même si j'avais toujours en tête que le but de ce travail, c'était ces gens. (...) Donc, après quelques mois, c'était facile pour moi de voir une femme enceinte allongée par terre et de ne rien ressentir. Donc, de ce point de vue, je peux comprendre, même si je ne peux pas l'accepter moralement et politiquement, pourquoi un flic est un flic, comment c'est possible qu'un soldat tue. Je peux comprendre comment les conditions te changent, comment l'environnement change la manière dont tu te comportes dans ce monde 65 ».
\end{abstract}

Dans un contexte de violations des droits quasi structurelles, les acteurs associatifs sont conduits par leurs missions à adopter un prisme utilitariste où, en dépit de leurs intentions professionnelles et/ou politiques, les étrangers retenus finissent souvent par être perçus comme des cas potentiellement porteurs d'une dénonciation plus large. Ce processus biaise le rapport aux humiliations du quotidien : en dénonçant certaines situations comme inadmissibles, les intervenants sont conduits à délimiter un seuil en dessous duquel les autres sont jugées acceptables ${ }^{66}$.

Non seulement les intervenants se trouvent ainsi endurcis, «blindés » en quelque sorte par la rétention, mais leurs conditions de travail entravent par ailleurs les velléités de communication vers l'extérieur, redoublant les murs du centre d'un "mur de silence », comme l'a nommé une intervenante de Moria ${ }^{67}$. L'ensemble du personnel est en effet soumis à un devoir de réserve plus ou moins explicite, les organisations étant dépendantes des autorités pour accéder aux lieux voire, pour une partie d'entre elles, liées à l'État par des contrats renouvelables. À Pagani par exemple, l'accès des associations locales au centre étant laissé à la discrétion de la police, les rares groupes autorisés doivent modérer leurs critiques au risque de ne pas voir leur autorisation renouvelée. Cette dépendance s’institutionnalise à Moria : si la loi 3907/2011 prévoit l'accès de la société civile aux lieux d'enfermement, la plupart des contrats de travail exigent la signature d'une clause de confidentialité qui vient formaliser l'interdiction de communiquer des informations internes. Comme dans le cas français décrit par Nicolas Fischer ${ }^{68}$, le dispositif s'officialise en

65. Entretien avec un ancien employé de Pagani, novembre 2013, Mytilène, LT.

66. Fischer N., « Jeu de regards. Surveillance disciplinaire et contrôle associatif dans les centres de rétention administrative », op. cit., pp. 45-65.

67. Entretien avec une employée de Moria, novembre 2013, Mytilène, LT. 
intégrant sa propre critique à son fonctionnement, mais en gardant la mainmise sur cette dernière. En outre, la culture de la discrétion est d'autant plus forte que les employés se trouvent souvent dans des situations précaires (missions de quelques mois ou CDD n'excédant pas un an dans le cadre de programmes européens) qui limitent leur liberté de parole ${ }^{69}$. Un ancien migrant enfermé à Pagani, qui était l'un de nos informateurs privilégiés en 2010, a ainsi refusé de témoigner sur Moria où il était employé comme interprète en 2013 de peur de mettre en jeu son emploi. "Certains travailleurs sociaux ont fait des rapports mais ce n'est qu'une fois leur mission au centre terminée qu'ils les ont fait parvenir aux ministères concernés 70 » confie une intervenante de Pagani, d'où l'intérêt de mener également des entretiens avec d'anciens employés.

Ces entraves n'empêchent pas pour autant les contestations au sein des lieux de rétention mais ces dernières, conditionnées par ce cadre contraignant, prennent plutôt la forme de «tactiques » qui « font avec 71 » l'institution en détournant certaines de ses règles (distribuer discrètement plus de vêtements que prévu, apporter des livres ou des jeux alors que c'est interdit, discuter avec des retenus quand l'inverse est recommandé, etc.). Elles reposent plus rarement sur des oppositions assumées, ou alors sous l'impulsion de certains intervenants particulièrement engagés, dotés de ressources spécifiques ou d'une position professionnelle plus stable. À Moria par exemple, à l'automne 2013, une employée de l'équipe médicale très active dans la défense des étrangers s'est opposée à un officier de police qui entendait priver de repas un Syrien ne s'étant pas présenté au comptage journalier des retenus. D'abord isolée et ignorée par l'officier, elle réussit à mobiliser ses collègues le lendemain lorsque ce dernier s'apprête, de nouveau, à sanctionner une femme âgée qui ne s'est pas rendue dans la cour pour être comptée parce qu'elle avait du mal à se déplacer. L'intervenante parvient à gagner l'appui du médecin, qui va s'avérer décisif : il contacte le siège de l'équipe médicale à Athènes qui informe la direction de la police, laquelle interdit à l'officier d'administrer ce type de sanctions dangereuses sur le plan médical. Le déroulement de cet épisode est emblématique des difficultés à se mobiliser en rétention et des marges de manœuvre dont disposent toutefois les intervenants, comme en témoigne l'employée en question :

68. Fischer N., « Jeu de regards. Surveillance disciplinaire et contrôle associatif dans les centres de rétention administrative ", op. cit.

69. Il est d'ailleurs intéressant de noter que le recours aux CDD vaut aussi pour les employés du ministère : bien que la loi 3907/2011 prévoie le recrutement du personnel des KEPY en contrat à durée indéterminé (article 9 paragraphe 3), une clause permet en cas « d'imprévu et de besoin urgent dû à un afflux massif d'immigrés, le recrutement du personnel selon l'article 20 de la loi 2190/1994 », accord-cadre sur le travail en contrat à durée déterminée (article 9 paragraphe 6).

70. Entretien avec une ancienne employée de Pagani, février 2010, Mytilène, LP.

71. De Certeau M., L'invention du quotidien. L'art de faire, Paris, Gallimard, 1990 (1980). 
«Je pense que quand on parle... parce que dedans il y a aussi la peur... et quand tu commences à parler, d'autres personnes vont aussi oser parler et ça crée quelque chose de différent, ça donne plus de force. Le deuxième jour, avec la femme, on était tous là et on lui a dit [à l'officier de police] : “ce n'est pas raisonnable ce que tu fais !" Mais d'habitude, parce que j'ai l'expérience de ça, quand tu es dans un centre, il y a l'autorité de la police et c'est accepté. Personne ne parle, même si tu dis que ça ne va pas, personne ne parle (...) Quand je suis allée voir [la médecin] pour le Syrien [privé de nourriture par le chef de police] par exemple, elle m’a dit: “moi, en tant que médecin, je dis que ce n'est pas acceptable”. Mais elle ne l'avait pas fait spontanément. Dans l'ensemble, il y a une sorte de silence pour ne pas être mis dehors 72 ».

Si certaines pratiques sont critiquées par les acteurs de la rétention, les contestations sourdes ne se transforment en protestation qu'à certaines conditions, notamment la présence d'un ou de plusieurs intervenants susceptibles, en fédérant les voix discordantes, d'empêcher l'auto-censure et de renverser le rapport de force. Dans ces conditions, le travail en rétention devient pour certains intervenants une forme de contestation en soi, une activité subversive par sa seule existence dans un contexte d'indifférence ou de violence, même s'il peut entrer en contradiction avec des positionnements éthiques ou politiques personnels. L’employée poursuit ainsi :

\begin{abstract}
«Pour moi c'est très dur d'être dedans, et en même temps, en regardant les réfugiés, je me dis que c'est très important. Même si au niveau politique je ne suis pas pour améliorer les conditions, pour eux, par exemple pour les Syriens, c'est très important qu'on soit là. Un Syrien me disait l'autre jour: "Mais c'est quoi ça ? Où sont les droits de l'homme ?” Et je lui ai dit : “Tu as raison, tu as raison”. Et même si je n'ai pas changé grand chose, au moins il n'est pas seul. Je pense qu'au niveau humain, c'est important d'avoir quelques personnes qui les considèrent d'une manière différente 73 ».
\end{abstract}

Refuser de travailler en rétention par principe, intervenir en privilégiant l'aide au quotidien, intégrer le dispositif pour le miner de l'intérieur, en faire partie tout en exprimant son désaccord : si l'éventail des positions est large et nuancé, les intervenants rencontrés reconnaissent tous, explicitement ou à demi-mots, être confrontés à des tensions et des contradictions plus ou moins difficiles à surmonter. Le travailleur social cité à propos de Pagani a par exemple fini par donner sa démission, seule solution possible à ses yeux pour retrouver sa liberté d'expression.

72. Entretien avec une employée du service médical de Moria, novembre 2013, Mytilène, LT.

73. Entretien avec une employée du service médical de Moria, novembre 2013, Mytilène, LT. 
Enfin, les ONG ont tendance à recruter certains salariés sur le continent au détriment des employés locaux ${ }^{74}$, ce qui contribue à isoler les centres de leur environnement proche. À Moria, en 2013, le service de premier accueil est composé uniquement de fonctionnaires envoyés d'Athènes et, au total, moins d'un quart des membres du personnel (policiers exceptés) est originaire de l'île. Les salariés du continent vivent par ailleurs souvent dans des résidences louées collectivement qui renforcent l'entre-soi. Enfin, la présence d'organisations internationales comme le HCR ou l'OIM, jugée suffisante par les autorités, vient légitimer des refus d'accès aux associations de l'île. Alors que l'organisation informelle de Pagani, qui autorisait certains arrangements avec la règle, permettait l'intervention ponctuelle de certains acteurs associatifs, la loi prévoyant l'accès des ONG aux centres de rétention (3907/2011) entretient de facto une distance à la société locale. Dans ce contexte, les militants locaux ont, à plusieurs reprises, cherché à développer des alternatives à la détention.

\section{Construire des alternatives et les défendre, à quel prix?}

L'absence chronique de lieux de rétention et le manque de structure appropriée pour les mineurs a entrainé à Lesbos le développement d'alternatives locales à l'enfermement. Bien que ces dernières aient été construites en opposition avec les logiques de contrôle, financées par des fonds distincts et gérées par des acteurs de la société civile, elles n'ont cessé d'entretenir un rapport ambivalent avec les dispositifs de confinement.

En juillet 2008, la «villa Azadi » est ouverte comme alternative au centre de Pagani qui enregistrait à l'époque une affluence record (figure 4). Fruit d'une action concertée à différentes échelles (militants locaux, ministère de la Santé et HCR), elle permet d'accueillir pour quelques jours ou plusieurs mois des mineurs transférés d'un centre de rétention ou venus de leur plein gré faute d'avoir pu quitter la Grèce.

"Les jeunes sont libres de rester ou de partir, c'est à leur guise » explique une employée de la Villa. "Certains attendent de la famille qui doit les rejoindre avant de partir, d'autres se reposent le temps de récupérer des forces pour la suite. Parfois ils partent vers Athènes et Patras et, ne pouvant passer la frontière, en avion ou par l'Italie, ils reviennent, se reposent et repartent ${ }^{75}$ ».

Censée être un lieu ressource au service des jeunes, la Villa est cependant rapidement prise dans une logique équivoque d'attractivité des mineurs dont la présence conditionne l'obtention de financements et donc le maintien de la

74. Les offres de poste doivent en effet être publiées au niveau national (loi 3907/2011) et circulent en interne, ce qui réduit sans l'empêcher la probabilité d'embauche de salariés de l'île extérieurs à l'organisation.

75. Entretien avec une employée de la Villa Azadi, avril 2009, Aghiassos, LP. 
structure. Afin de pousser les jeunes à rester dans le lieu pour pérenniser son existence, la direction leur offre notamment la possibilité de gagner quelques euros en exerçant des missions rémunérées à la journée par des agriculteurs qui trouvent là une main d'œuvre bon marché. Si l'établissement est initialement géré par une équipe de jeunes travailleurs sociaux engagés en faveur de l'insertion des mineurs, des changements de direction dus à une interruption du programme, prévu pour deux ans ${ }^{76}$, vont peu à peu transformer l'équipe et le mode de fonctionnement du lieu. L'enjeu est de parvenir à retenir les mineurs sans les détenir : les intervenants cherchent, tout en les laissant libres, à 《 mettre en place des activités pour ne pas les faire partir 77 » :

« [Les enfants] ont besoin de croire en nous, c'est le nouveau challenge. On leur donne de l'amour et on leur montre qu'on n'est pas là pour leur faire du mal, mais en même temps on met des barrières. En ce moment il y a 53 enfants et 13 employés. Les plus petits on essaie de les garder près de nous parce qu'ils sont vraiment fragiles et potentiellement en danger, à Athènes notamment. Sur 38 enfants qui sont arrivés d'Evros la semaine dernière il n'en reste que 20 aujourd'hui. Nous ne voulons pas les laisser libre dans la mesure où ils sont trop en proie à des dangers 78 ».

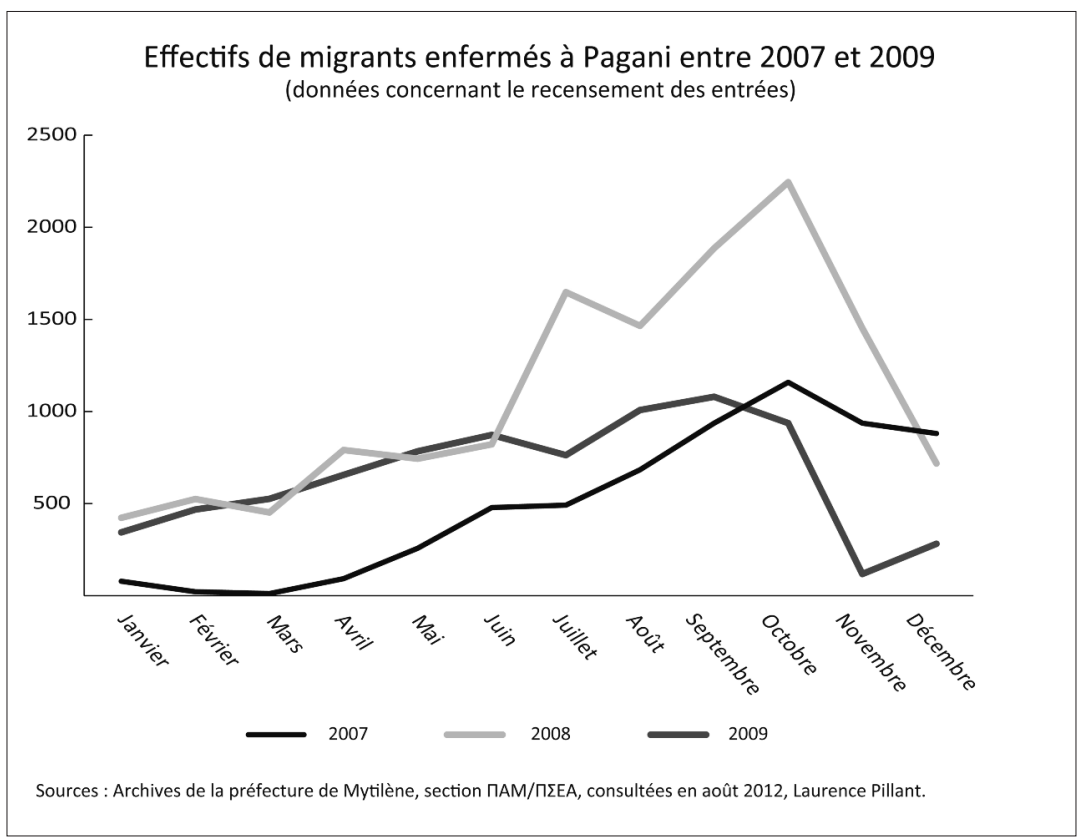

Figure 4

76. Faute de financements, les activités et les emplois à la «Villa Azadi » ont été interrompus durant plusieurs mois entre juillet 2010 et janvier 2011 puis à partir d'avril 2013.

77. Responsable de la Villa Azadi, février 2011, Aghiassos, LP.

78. Entretien avec une employée du service médical à la Villa Azadi, février 2011, Aghiassos, LP. 
En dépit de débats internes, les règles régissant l'établissement se durcissent (appels de présence réguliers et visites davantage contrôlées) et contribuent à la relégation progressive des jeunes. Situé en pleine montagne à $4 \mathrm{~km}$ $\mathrm{du}$ village le plus proche et à $40 \mathrm{~km}$ de la capitale, cet ancien sanatorium devient doublement synonyme d'isolement pour les mineurs, qui sont physiquement et symboliquement tenus à distance de la ville et de ses habitants.

Dans ce contexte, l'établissement est récupéré politiquement par les acteurs du contrôle. Erigé en exemple par les autorités de police et les institutions insulaires qui mettent en avant le volet « humanitaire » du système envers les populations dites "vulnérables 79 », il devient à la fin des années 2000 un nœud du dispositif où tous les mineurs isolés de la région sont transférés sans que leur accompagnement et leur suivi à long terme ne constituent pour autant une réelle priorité. En ce sens, la Villa Azadi n'est pas sans rappeler le fonctionnement aléatoire du contrôle migratoire sur l'île. Tributaire des financements européens, elle n'a cessé d'être fermée puis rouverte au gré des contrats publics. En 2013, fermée du jour au lendemain pendant six mois faute de subventions, elle est ainsi vidée de ses salariés sans qu'aucune proposition de relogement ne soit faite à ses occupants. Abandonnés à eux-mêmes et à la solidarité des habitants, dont certains s'organisent pour leur apporter des repas via le directeur du centre, une vingtaine de jeunes rencontrés en août 2013 disaient avoir le sentiment de «devenir fou » dans ce qu'ils appelaient la «prison de la montagne 80 ».

Depuis 2012, Lesbos a par ailleurs été la scène d'une initiative plus radicale et relativement inédite aux frontières de l'Union européenne : l'organisation d'un camp d'accueil autonome alternatif à la détention. Alors que les étrangers arrivés à partir d'août 2012 étaient détenus dans le port ou laissés à la rue, des militants ont décidé d'investir une base de loisirs située à environ huit kilomètres de la capitale pour y accueillir les migrants. Fédérant autour d'eux des dizaines d'habitants aux profils très hétérogènes - des activistes anarchistes à la bourgeoisie chrétienne en passant par des cadres de partis de gauche -, le «Village of all together 81 » a fonctionné en novembre 2012 puis ponctuellement en fonction des arrivées. L'histoire de ce site, prénommé PIKPA, entre en résonance avec celle de la rétention : détourné de son usage ordinaire, il a été utilisé chroniquement en parallèle des espaces officiels d'enfermement dans les années 1990, durant l'été 2009 puis à partir de l'hiver 2012.

Son utilisation, conditionnée à l'autorisation de la municipalité, est le fruit d'un compromis qui répond à des intérêts convergents. Du côté des autorités, l'existence d'un lieu de prise en charge pour lequel elles n'ont rien à dépenser ni organiser représente une aubaine. Non seulement le «Village » offre aux

79. Entretien avec le Préfet de Lesbos, février 2010, Mytilène, LP.

80. Entretien avec des jeunes de la Villa Azadi, août 2013, Aghiassos, LT.

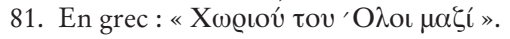


étrangers des conditions d'accueil que les policiers et les garde-côtes n'étaient pas en mesure de leur garantir, mais il les rassemble par ailleurs dans un lieu circonscrit éloigné des regards. Du côté des organisateurs, la mise à disposition d'un espace collectif équipé fournit une base matérielle pour concrétiser leur projet. En dépit de plusieurs problèmes (ressources limitées et difficultés à mobiliser sur le long terme), l'initiative attire des migrants, des militants et des artistes ${ }^{82}$ qui s'installent dans le camp et/ou s'y investissent, montrant qu'une solution alternative à l'enfermement, plus respectueuse des droits humains, moins coûteuse et tout autant efficace d'un point de vue policier (les étrangers ne cherchent pas à fuir comme ils ont besoin d'une obligation à quitter le territoire), est possible.

Le projet rencontre néanmoins plusieurs limites. La municipalité, d'abord, semble tolérer l'initiative à condition qu'elle lui soit utile et ne lui porte pas préjudice. Fin novembre 2012, alors que le projet connaît une médiatisation croissante après un mois de fonctionnement et attire de nombreux journalistes, la police délivre des obligations à quitter le territoire à tous les migrants installés à PIKPA, entraînant leur départ collectif pour Athènes. Les autorités rappellent ainsi qu'elles gardent la mainmise sur les étrangers qu'elles peuvent, si ce n'est bloquer, au moins faire circuler. Plus directement, les garde-côtes essayent au printemps 2013 d'imposer la présence de l'un d'eux en permanence, ce qui divise profondément le «Village », une partie refusant de gérer un « lieu d'enfermement qui ne dit pas son nom ${ }^{83}$ ».

En l'absence de compromis, PIKPA est abandonné et ses derniers membres se remettent à assister les migrants dans le port en leur apportant des repas. L'ouverture du centre de Moria en septembre 2013 relance le projet mais en transforme les modalités, les policiers décidant d'envoyer à PIKPA les migrants libérés du centre qui doivent attendre le départ des ferries (deux à trois fois par semaine en hiver) sans soutenir pour autant le «Village». En 2013 et 2014, si le camp permet d'accueillir une population affaiblie par la rétention et représente pour elle la première étape d'un nouveau parcours, il n'empêche donc pas leur enfermement et contribue même au bon fonctionnement du centre où des places sont ainsi libérées pour «accueillir » de nouveaux arrivants.

Pour conclure, s'intéresser aux ressorts et aux effets du contrôle migratoire sur l'île de Lesbos montre que le discours du blindage frontalier affiché par les institutions européennes, par l'agence Frontex et par les gouvernements des États membres s'effondre à l'épreuve des faits. À Lesbos, les frontières ne sont pas infranchissables mais poreuses, et les arrivées de migrants

82. La frontière entre ces catégories est poreuse, certains migrants ayant eu une expérience militante ou artistique et plusieurs activistes ou artistes impliqués à PIKPA étant eux-mêmes étrangers.

83. Entretien avec un ancien membre du «Village », août 2013, Mytilène, LT. 
continuent en dépit des opérations de contrôle sans même être anticipées par les autorités, ce que démontrent une fois de plus les événements de l'été 2015. Le recours à l'enfermement, tout en étant systématique, relève moins de procédures établies que de pratiques policières discontinues exercées dans des lieux hybrides et dans l'improvisation, les migrants se trouvant tour à tour enfermés dans des espaces clos et des formes de circulation contrainte. Les solutions sécuritaires, enfin, ont précisément conduit la société civile à développer des alternatives à ce qui constitue l'outil essentiel du blindage - la rétention administrative -, impulsant plusieurs projets de solidarité qui opposent à l'enfermement et à l'isolement des espaces d'accueil et d'échanges. Cependant, en contribuant aux évolutions des dispositifs de contrôle, ces initiatives ont elles-mêmes eu un certain effet «blindant » sur les habitants qui, sauf situation exceptionnelle (été 2009, automne 2012 et été 2015), sont tenus à distance des lieux d'enfermement et, pour ceux qui y travaillent, confinés dans un certain silence.

Aussi, fait de tâtonnements et d'ambivalences, le cas de Lesbos conduit à décentrer le regard porté sur les lieux de rétention pour s'intéresser à la manière dont ces espaces et leur environnement sont mutuellement travaillés l'un par l'autre. En montrant à quel point le dispositif évolue au gré des mobilisations de la société civile, qui s'en trouve elle-même mise à l'épreuve, il invite à s'intéresser davantage, à l'instar de plusieurs travaux récents, à la " continuité entre l'intérieur et l'extérieur des murs ${ }^{84}$ » de l'enfermement. Non seulement le dispositif de contrôle se caractérise par une myriade de lieux qui dépassent largement l'enceinte des centres officiels et constituent, dans certains cas, une passerelle vers le continent, mais ces derniers s'inscrivent même dans un continuum avec les espaces alternatifs qui, en reproduisant certaines modalités de confinement, viennent eux aussi faire frontière. Toutefois, bien que fragiles et traversées par des contradictions, les mobilisations menées localement offrent aussi, par leurs résultats inédits, d'autres horizons politiques à même de questionner la légitimité de l'enfermement des étrangers. 


\section{Encadré méthodologique Enquêter sur l'enfermement des migrants à Lesbos}

Fondé sur deux recherches en sociologie et en géographie menées sur l'île entre 2009 et 2014, cet article est issu de la rencontre entre deux travaux de doctorat qui questionnent les nouvelles formes frontalières auxquelles sont confrontés les migrants dans l'UE. Les deux auteures ont mené leurs enquêtes à des périodes distinctes, privilégiant des retours répétés sur le terrain allant de quelques jours à plusieurs mois, chaque année, entre le printemps 2009 et mai 2014. Ces périodes successives ont permis de mener un travail d'archive dans la préfecture de l'île en charge de la gestion des lieux d'enfermement et d'effectuer des observations participantes via des membres de la société civile dans le port, le commissariat et les centres de rétention de Pagani et de Moria. Au total, une centaine d'entretiens ont été menés en grec, en anglais ou en français auprès des acteurs du contrôle migratoire (policiers, garde-côtes, agents Frontex, fonctionnaires locaux en charge des questions de migration), auprès d'élus politiques de la majorité et de l'opposition, d'intervenants dans les lieux de rétention (interprètes, employés d'ONG ou d'organisations internationales, agents de restauration et de nettoyage), d'habitants vivant à proximité des lieux d'enfermement, d'entrepreneurs impliqués dans la construction ou la maintenance des bâtiments, de militants locaux et internationaux actifs sur l'île et, enfin, de personnes en situation de migration rencontrées au cours ou à l'issue de leur maintien en rétention. Ce travail à Lesbos a été complété par des interviews à Athènes auprès de migrants, de responsables de police, $\mathrm{d}^{\prime} \mathrm{ONG}$ et d'organisations internationales et par un travail d'archive au ministère de I'Intérieur. Un corpus rassemblant des articles de la presse locale sur les questions de migrations a été peu à peu élaboré et enrichi par des articles consacrés à la frontière grécoturque dans la presse internationale.

L'enquête a été menée dans un contexte national difficile, marqué par de profondes transformations sociales et par la montée en puissance des mesures sécuritaires à l'égard des migrants : cinq changements de gouvernement en six ans, une crise économique sans précédent, l'accession au parlement d'un parti néo-nazi, la mise en place de plusieurs opérations contre l'immigration clandestine avec et sans le soutien de Frontex, l'ouverture d'une dizaine de lieux d'enfermement et la construction d'une barrière à la frontière gréco-turque. Dans ce contexte, mener une enquête ethnographique sur la rétention des étrangers à Lesbos confronte le chercheur à un objet contradictoire à plusieurs niveaux.

Premièrement, si les arrivées de migrants sont régulièrement placées sous le feu des projecteurs (débarquements médiatisés, érigés en symbole des migrations clandestines dans I'UE et exploités politiquement à l'échelle locale et nationale), leur gestion est tenue dans l'opacité (absence de procédures claires, informations délivrées au compte-goutte, lieux de confinement difficiles d'accès, etc.). Entre sur-exposition du phénomène et pratiques d'enfermement obscures, I'enquête a donc dû procéder par tâtonnements dans un travail conjoint de déconstruction du "spectacle de la frontière 85 » et de reconstitution, parfois épineuse, des mécanismes de contrôle.

Deuxièmement, la rétention constitue un objet d'étude matériellement délimité donc a priori aisé à définir (un dispositif d'enfermement circonscrit par ses murs) mais ses contours à Lesbos sont en fait incertains (des pratiques fluctuantes et peu réglementées dans des espaces disparates). L'enjeu était alors de surmonter les différents obstacles entravant l'accès à ces lieux sans pour autant les réduire à des îlots de réclusion détachés de leur environnement - comme ces difficultés auraient pu y amener.

85. Cuttitta P., Lo spettacolo del confine, Mimesis, Milan, 2012, 127 p. 
Troisièmement, Lesbos est un territoire insulaire avec un réseau d'acteurs resserré, ce qui se prête particulièrement à l'enquête ethnographique, mais l'île est à certaines périodes saturée de professionnels de la migration : journalistes, organisations internationales, étudiants ou chercheurs. Ces acteurs sollicitent en général les mêmes interlocuteurs, dont les contacts circulent, favorisant la construction de discours types et de parcours obligés sur I'île (le port, la villa Azadi, le cimetière, le centre de rétention, PIKPA...) qu'il importe à la fois d'analyser et de contourner. Notre présence sur la durée au cours de séjours répétés, y compris hors des périodes de médiatisation de l'île, a été un atout en ce sens.

À ces difficultés s'en ajoute une dernière, à la fois plus intime et plus générale : la gestion personnelle de la confrontation, parfois quotidienne, à des situations de violence et/ou de détresse. L'enquête en rétention et les échanges informels avec des personnes enfermées ont été souvent éprouvants, tant dans les situations observées et les témoignages recueillis que dans la position inconfortable dans laquelle nous étions, suscitant malgré nos explications des attentes matérielles ou politiques - servir de porte-voix - que nous ne pouvions pas ou peu satisfaire. A l'image du processus que nous observions, nous avons été ainsi conduites à nous « blinder » partiellement vis-à-vis de nos émotions : si nous avons cherché à les mettre à distance et à les objectiver afin de ne pas être prises au piège d'une interprétation « à chaud » des phénomènes, nous n'avons cependant jamais essayé de nous en prémunir, pensant au contraire qu'il était important d'y prêter attention afin de ne pas céder à la normalisation de la violence produite par le contrôle migratoire, laquelle est un ressort de sa dépolitisation. Aussi les entretiens individuels ont-ils été menés en majorité à l'issue du maintien en rétention dans des lieux propices à la discussion, notamment le camp alternatif de PIKPA. Ce cadre ne faisait bien sûr pas disparaître les frontières sociales nous séparant de nos interlocuteurs, en particulier le décalage en matière de droit à la circulation, mais il permettait néanmoins d'éviter l'asymétrie des relations produites par l'enfermement.

Dans ce contexte propice aux jugements moraux et aux interprétations normatives - un terrain médiatique, des questions politiquement sensibles, des personnes en souffrance, des lieux à l'abri des regards -, les questions méthodologiques ont occupé une place centrale. Le cheminement de nos enquêtes est d'ailleurs significatif, reflétant à bien des égards les dysfonctionnements du système de contrôle et ses effets sur la société locale.

Nous avons sollicité les autorités nationales et locales à plusieurs reprises pour obtenir l'autorisation de mener nos recherches dans les lieux de rétention, mais nous n'avons reçu aucune réponse. Les demandes officielles n'ayant pas abouti, il a donc fallu se débrouiller sur place au jour le jour : identifier les responsables, toquer aux portes, faire jouer les réseaux $d^{\prime}$ 'interconnaissance, multiplier les interlocuteurs, gagner leur confiance, re-toquer aux portes... et c'est finalement cette démarche informelle, peut-être plus adaptée au fonctionnement relativement souple des autorités locales, qui a fait avancer l'enquête. Si notre présence a parfois suscité la méfiance (avec des contrôles d'identité à la limite de l'interrogatoire) ou au contraire un intérêt déplacé (lié à notre genre et à notre statut), les entretiens avec la police, les garde-côtes et les agents préfectoraux ont dans l'ensemble permis d'obtenir des données et des informations précieuses, en particulier chez des agents subalternes en l'absence de leur hiérarchie.

L'accès aux lieux de rétention ne nous pas été accordé en dépit des contacts noués, ce qui a constitué un obstacle majeur mais paradoxalement édifiant : non seulement il nous a confrontées aux barrières bureaucratiques protégeant la rétention des regards extérieurs, éclairant en cela certains ressorts du contrôle, mais il nous a aussi fait prendre une distance salutaire avec notre objet. En nous poussant à interroger des personnes liées de près ou de loin aux lieux d'enfermement (y travaillant, habitant à proximité ou se mobilisant sur cette 
question) faute de pouvoir y accéder par nous-mêmes, l'absence d'autorisation a déplacé notre regard vers ce qui se passait autour de la rétention. Notre enquête s'est ainsi éloignée du dispositif pour mieux le retrouver. D'une part, ce détour nous a permis d'appréhender les lieux de rétention dans leur globalité, c'est-à-dire comme un ensemble d'espaces divers en relation avec la société locale ; d'autre part, il nous a offert une voie d'entrée alternative en leur sein via les réseaux associatifs.

Notre accès aux lieux d'enfermement résulte en effet de la conjonction entre la gestion peu formalisée de la rétention (notre identité n'a par exemple jamais été contrôlée à l'entrée des sites) et la mobilisation de membres de la société civile - volontaires dans le port, ONG à Moria, association locale à Pagani - qui nous ont offert la possibilité de les accompagner. Si cette entrée sur le terrain n'a pas toujours laissé la latitude espérée pour nos enquêtes, notre position de chercheuse étant plus ou moins connue selon les périodes, elle nous a permis d'observer à la fois la vie quotidienne en rétention, le travail ambivalent d'assistance en son sein et les projets alternatifs développés à l'extérieur, certains intervenants étant aussi actifs dans les milieux militants. Les rencontres et les amitiés nouées à cette occasion ont enfin renforcé le sens de notre travail (guidé par la volonté de briser l'évidence du recours à la rétention par l'étude de ses mécanismes et de ses effets) en donnant lieu à des discussions à bâtons rompus, à des actions collectives et à des festivités improbables, autant de moments d'échanges et de confrontations qui fondent aussi la recherche. 